\title{
GENERALIZED DIMENSION ESTIMATES FOR IMAGES OF POROUS SETS IN METRIC SPACES
}

\author{
Aapo Kauranen \\ University of Jyväskylä, Department of Mathematics and Statistics \\ P. O. Box 35 (MaD), FI-40014 University of Jyväskylä, Finland; aapo.p.kauranen@jyu.fi
}

\begin{abstract}
In $Q$-Ahlfors regular metric measure spaces we obtain essentially sharp dimension estimates for images of porous sets under Hajłasz-type Sobolev maps with $L^{Q}$-gradient. The estimates generalize the previous results of Jones and Makarov [7] and Kauranen and Koskela [8].
\end{abstract}

\section{Introduction}

In 1995 Jones and Makarov [7] showed that every conformal map $f$, defined in the unit disk maps the unit circle to a set of zero Lebesque measure, provided $f$ has a modulus of continuity $\psi$ satisfying

$$
\int_{0}\left|\frac{\log \psi(t)}{\log t}\right|^{2} \frac{d t}{t}=\infty .
$$

Moreover, their divergence condition is sharp in the sense that given a $\psi$ such that the above integral converges, it is possible to construct a conformal map, with modulus of continuity $\psi$, which maps the unit circle onto set of positive Lebesque measure. The condition (1) means that the integral in question is infinite for intervals $[0, c]$, for any $c>0$. That is, the integral diverges at zero.

Recently Kauranen and Koskela [8] generalized this result to the setting of the Sobolev space $W^{1, n}(B(0,1))$ with modulus of continuity $\psi$ satisfying

$$
\int_{0}\left|\frac{\log \psi(t)}{\log t}\right|^{n} \frac{d t}{t}=\infty
$$

Their method also yields generalized Hausdorff dimension estimates which were shown to be sharp for Hölder continuous maps.

The purpose of this paper is to show that the methods used in [8] can be applied in the setting of $Q$-Ahlfors regular metric measure spaces and that the obtained generalized Hausdorff dimension estimates are essentially sharp for each $Q>1$ and each modulus of continuity satisfying (3). Our main result is the following.

Theorem 1.1. Let $Y$ be a metric space, $X$ be a $Q$-Ahlfors regular metric measure space, with $Q>1$, and $E \subset X$. Assume that $E$ is closed and porous. Suppose that $f: X \rightarrow Y$ has an $L^{Q}$-Hajłasz gradient $g$ and is continuous with an allowable modulus of continuity $\psi$ satisfying

$$
\int_{0}\left|\frac{\log \psi(t)}{\log t}\right|^{Q} \frac{d t}{t}=\infty .
$$

doi:10.5186/aasfm.2016.4107

2010 Mathematics Subject Classification: Primary 30L99, 26B35, 28A78.

Key words: Metric spaces, Sobolev spaces, Hausdorff measure, modulus of continuity.

The author has been supported by the Academy of Finland via the Centre of Excellence in Analysis and Dynamics Research (project No. 271983). The paper is a part of the author's PhD thesis. 
Then $\mathcal{H}^{Q}(f(E))=0$. Moreover, $\mathcal{H}^{h}(f(E))=0$ for all gauge functions $h$ of the form $h(t)=t^{Q} \eta(t)$, where $\eta$ is any decreasing continuous function satisfying

$$
\eta\left(2^{-k}\right) \leq \sum_{j=1}^{k}\left(\frac{\psi^{-1}\left(2^{-j}\right)}{2^{-j}\left(\psi^{-1}\right)^{\prime}\left(2^{-j}\right)}\right)^{Q-1}
$$

for all $k \in \mathbf{N}$.

The necessary definitions are given in the following section. Theorem 1.1 recovers the result from [8]: $\partial B(0,1)$ is porous and the set of the continuous maps $f: B^{n}(0,1) \rightarrow \mathbf{R}^{m}$ in $W^{1, n}\left(B^{n}(0,1), \mathbf{R}^{m}\right)$ have $L^{n}$-Hajłasz gradients (see [4, Theorem 6.10]). Theorem 1.1 is a corollary to a more abstract statement proved in Section 3. The proof of Theorem 1.1 is given in Section 4.

Our second result shows the sharpness of (4) in Theorem 1.1.

Theorem 1.2. Let $n \geq Q>1$ and $\beta>1$. Let $\psi$ be an allowable modulus of continuity satisfying (3) and $\psi(t) \geq t^{a}$, with $a<\frac{1}{n}$, for all small $t$. There exists a $Q$-Ahlfors regular metric measure space $X$ and a closed porous set $E \subset X$ and a mapping $f: X \rightarrow \mathbf{R}^{n}$, with an $L^{Q}$-Hajłasz gradient and modulus of continuity $C \psi$, for some $C>0$, such that $\mathcal{H}^{h}(f(E))>0$, for an increasing gauge function satisfying

$$
h\left(2^{-k}\right)=2^{-k Q}\left(\sum_{j=1}^{k}\left(\frac{\psi^{-1}\left(2^{-j}\right)}{2^{-j}\left(\psi^{-1}\right)^{\prime}\left(2^{-j}\right)}\right)^{Q-1}\right)^{\beta}
$$

for all $k \in \mathbf{N}$.

The condition $\psi \geq t^{a}$ cannot be completely removed, since when $Q=n$ the claim says that the Hausdorff dimension of $f(E)$ should be $n$. This cannot be achieved if the Hölder exponent is large enough, because porous sets have always strictly smaller Hausdorff dimension than the ambient space. Modifying the map $h$ of Section 4 in [8] shows that Theorem 1.2 is true even if $a=\frac{1}{n}$, but there the construction is different and done only in Euclidean spaces.

The example referred to in Theorem 1.2 is constructed in Section 5. The proof of Theorem 1.1 is based on a variant of mean porosity arguments developed in [10], [13] and [8]. The example showing the sharpness is a modification of the example given in [9].

Acknowledgements. The author wishes to thank his advisor Professor Pekka Koskela for suggesting the problem and reading the manuscript, and anonymous referee for numerous comments which improved the exposition and noting a mistake in earlier version of Section 5.2.

\section{Preliminaries}

2.1. Notation. Throughout the paper $C$ denotes a positive constant, possibly different from line to line, which may depend only on structural constants of spaces, approximating families, and constants in the modulus of continuity. Additional dependencies are listed inside parentheses as usual.

2.2. Some concepts in metric measure spaces. Throughout the paper $X$ and $Y$ denote metric spaces with metrics $d_{X}$ and $d_{Y}$ respectively. Most of the time we omit the subscript since the metric space in question is usually clear from the context. $B(x, r)$ denotes an open ball with center $x$ and radius $r$. If $B:=B(x, r)$ 
and $\alpha>0$ then $\alpha B=\alpha B(x, r)=B(x, \alpha r)$. Given a ball $B$ with radius understood $r(B)$ denotes the radius of this ball.

Recall that the set $E$ is called porous if there exist positive numbers $r_{0}$ and $\alpha$ such that for every $x \in E$ and every $0<r<r_{0}$ there exists another ball $B^{\prime}$ of radius $\alpha r$ such that $B^{\prime} \subset B(x, r) \cap X \backslash E$. A metric measure space $(X, d, \mu)$ is $Q$-Ahlfors regular $(Q>0)$ if there exist $C$ and $r_{0}>0$ such that

$$
C^{-1} r^{Q} \leq \mu(B(a, r)) \leq C r^{Q},
$$

for all $r<r_{0}$ and all $a \in X$.

Definition 2.1. Let $(X, \mu)$ be a metric measure space and $Y$ a metric space. Let $f: X \rightarrow Y$ be a mapping. A measurable function $g: X \rightarrow[0, \infty]$ is called a Hajłasz gradient of $f$ if there exists a set $A \subset X$ with $\mu(A)=0$ such that for all $x, y \in X \backslash A$

$$
d_{Y}(f(x), f(y)) \leq d_{X}(x, y)(g(x)+g(y)) .
$$

If a Hajłasz gradient $g$ belongs to the space $L^{p}(X), p \geq 1$, it is called an $L^{p}$-Hajłasz gradient.

A Hajłasz gradient does not directly correspond to the classical gradient but instead essentially to the maximal function of the gradient (see [3]). Another common choice for Sobolev spaces in metric spaces is the so-called Newtonian Sobolev space $N^{1, p}$. These spaces coincide with each other if the domain is a doubling metric measure space, with locally finite non trivial measure, supporting $(1, q)$-Poincaré inequality for some $p>q \geq 1$ (see [5, Cor. 9.1.10]).

2.3. Modulus of continuity. We say that a function $f: X \rightarrow Y$ has an allowable modulus of continuity $\psi$ if

$$
d(f(a), f(b)) \leq \psi(d(a, b)),
$$

for all $a, b \in X$ and $\psi$ is an allowable modulus of continuity in the sense of the following definition.

Definition 2.2. A continuously differentiable increasing bijection $\psi:(0, \infty) \rightarrow$ $(0, \infty)$ is an allowable modulus of continuity if there exists $t_{0}<1$ and $\beta>0$ such that for every $t \leq t_{0}$ the following conditions hold:

$$
\begin{aligned}
& \log \frac{1}{\psi^{-1}(t)} \text { is differentiable and } \frac{\left(\psi^{-1}\right)^{\prime}(t)}{\psi^{-1}(t)} t \text { is a decreasing function; } \\
& \log \frac{1}{\psi^{-1}(t)} \leq \beta \log \frac{1}{\psi^{-1}(\sqrt{t})} \\
& \frac{(\log \psi(t))^{\prime} t \log t}{\log \psi(t)} \text { is a monotone function. }
\end{aligned}
$$

The functions

$$
\psi(t)=C_{0} \exp \left(-C_{1} \log ^{s} \frac{1}{t}\right)
$$

where $C_{0}, C_{1}>0$ and $0<s \leq 1$, are examples of allowable moduli of continuity. More general examples can be found in [8].

2.4. Hausdorff measures. Let $h:[0, \infty) \rightarrow[0, \infty)$ be a gauge function, that is, a continuous non-decreasing function with $h(0)=0$ and $h(t)>0$ for all $t>0$. The generalized $h$-Hausdorff measure of a set $A \subset X$ is

$$
\mathcal{H}^{h}(A)=\lim _{\delta \rightarrow 0} \mathcal{H}_{\delta}^{h}(A),
$$


where

$$
\mathcal{H}_{\delta}^{h}(A)=\inf \left\{\sum_{U \in \mathcal{U}} h(\operatorname{diam} U): A \subset \bigcup_{U \in \mathcal{U}} U, \operatorname{diam} U \leq \delta\right\} .
$$

In our proofs we will need the following result of Davies [1, Section 8].

Lemma 2.3. Let $X$ be a metric space, $E \subset X$ and $h$ a gauge function satisfying $h(2 t) \leq C h(t)$ for some constant $C$ and all $t>0$. Then for any increasing sequence of sets $E_{n} \nearrow E$ and every $\delta>0$ we have $\mathcal{H}_{\delta}^{h}\left(E_{n}\right) \rightarrow \mathcal{H}_{\delta}^{h}(E)$ as $n \rightarrow \infty$.

Let $\left(c_{i}, U_{i}\right)_{i \in I}$ be a collection of pairs such that $c_{i}>0$ and $U_{i} \subset X$ for every $i \in I$, where $I$ is some countable index set. This collection is a weighted cover of the set $A$ if $\chi_{A} \leq \sum_{i \in I} c_{i} \chi_{U_{i}}$. The weighted Hausdorff content of the set $A \subset X$ is defined as

$$
\mathcal{H}_{w, \infty}^{h}(A)=\inf \left\{\sum_{i \in I} c_{i} h\left(\operatorname{diam} U_{i}\right):\left(c_{i}, U_{i}\right)_{i \in I} \text { is a weighted cover }\right\} .
$$

The following lemma states the relation between the weighted and unweighted Hausdorff measures.

Lemma 2.4. Let $h$ be a gauge function satisfying $h(2 t) \leq c h(t)$ for some constant $c>0$. Then for some $c_{h}>0$ we have $\mathcal{H}_{\infty}^{h}(E) \leq c_{h} \mathcal{H}_{w, \infty}^{h}(E)$ for each $E \subset X$.

The claim follows from [1, Section 8] and [2, 2.10.24], see also [6, Theorems 8.3 and 9.7]. For the case of compact $X$ the proof can be found in [12, Lemma 8.16].

To estimate Hausdorff dimensions we need the following Frosman's lemma.

Lemma 2.5. Let $B \subset \mathbf{R}^{n}$ be a compact set. Then $\mathcal{H}^{h}(B)>0$ if and only if there exists a Radon measure $\mu$ with $\operatorname{supp} \mu \subset B, \mu\left(\mathbf{R}^{n}\right) \in(0, \infty)$, and $\mu(B(x, r)) \leq h(r)$ for every $x \in \mathbf{R}^{n}$ and $r>0$.

For a proof see, for example, [12, Theorem 8.8].

\section{The core of the proof of Theorem 1.1}

In this section we prove a slight generalization of Theorem 1.1. We consider sets which can be approached in a controlled manner by "dyadic" balls. We have stated the assumptions in Proposition 3.2 in terms of the mapping and we do not have to assume any properties of the domain or the target. In the next section, we will show that porous sets can be approached in a suitable manner and functions with $L^{Q}$-Hajłasz gradients in $Q$-Ahlfors regular metric measure spaces satisfy the assumptions of Proposition 3.2.

Definition 3.1. Let $E \subset X$ be a set and let $j_{0} \in \mathbf{N}$. We call a countable family of balls $\mathcal{F}=\cup_{j=j_{0}}^{\infty} \mathcal{F}_{j}$ an approximating family of $E$ if the following conditions hold for some $C \geq 1$

(A1) $C^{-1} 2^{-j} \leq \operatorname{diam} B \leq C 2^{-j}$ for every $B \in \mathcal{F}_{j}$,

(A2) $\forall x \in E$ there exists sequence $\left(B_{j}\right)_{j=j_{0}}^{\infty}$, called an approximating sequence, such that $B_{j} \in \mathcal{F}_{j}$ and $d\left(B_{j}, x\right) \leq C 2^{-j}$ for all $j \geq j_{0}$,

(A3) there exists $N \in \mathbf{N}$ such that for all $x \in X$

$$
\sum_{B \in \mathcal{F}} \chi_{B}(x) \leq N
$$


If $\mathcal{F}$ is an approximating family then a collection of pairs $\left\{\left(x_{B}, B\right): B \in \mathcal{F}\right\}$, with $x_{B} \in B$, is a pointed approximating family. We use the notation $\mathcal{F}$ also for pointed approximating families.

Remark. (1) In the definition above the number $j_{0}$ acts as a starting level of the approximation. By changing the constant $C$ we could assume that $j_{0}=1$.

(2) All $\mathcal{F}_{j}$ are also countable.

Proposition 3.2. Let $1<Q<\infty$. Let $X$ and $Y$ be metric spaces. Let $E$ be a subset of $X$ with a pointed approximating family $\mathcal{F}$. Suppose that $f: X \rightarrow Y$ with an allowable modulus of continuity $\psi$ satisfies the conditions

(P1) for each $B \in \mathcal{F}$ there is $r_{B} \geq 0$ such that

$$
\sum_{j=0}^{\infty} \sum_{B \in \mathcal{F}_{j}} r_{B}^{Q}<\infty
$$

(P2) for every $x \in E$ the approximating sequence $\left(B_{i}\right)_{i=0}^{\infty}$ satisfies

$$
d\left(f\left(x_{B_{i}}\right), f\left(x_{B_{i+1}}\right)\right) \leq \min \left\{r_{B_{i}}, r_{B_{i+1}}\right\}
$$

for all $i \in \mathbf{N}$,

(P3) the function $\psi$ satisfies the divergence condition (3).

Then $\mathcal{H}^{h}(f(E))=0$, where $h$ is a gauge function as in Theorem 1.1.

Remark. In 3.2 the numbers $r_{B}$ do not refer to the radii of balls $B \in \mathcal{F}$. They are just non-negative real numbers, which are used as radii to define balls in the image of $f$.

Proof. Our proof is based on a covering argument similar to the ones in [10],[13] and [8]. In the calculations below we need to use (9) and (10) roughly speaking only for the tail ends of the approximating sequences. We may assume, possibly changing the number $j_{0}$ in the Definition 3.1, that all properties (9), (10) and (11) hold in all subsequent calculations.

We first fix some notation: define $\alpha(t)=\frac{\psi^{-1}(t)}{\left(\psi^{-1}\right)^{\prime}(t)}$ and $\lambda(k)=\frac{2^{-k}}{\alpha\left(2^{-k}\right)}$. The assumption (9) now implies that $\lambda$ is increasing for large $k$. Instead of working with (3), we will use the following condition equivalent to (3) (see [13, Remark 5.3])

$$
\int_{0}\left|\frac{\psi^{-1}(t)}{\left(\psi^{-1}(t)\right)^{\prime}}\right|^{Q-1} \frac{d t}{t^{Q}}=\infty .
$$

Given an arbitrary $\varepsilon>0$ we will show that $\mathcal{H}^{h}(f(E))<\varepsilon$. Condition 3.2 implies the existence of $m_{\varepsilon}$ such that

$$
\sum_{j=m_{\varepsilon}}^{\infty} \sum_{B \in \mathcal{F}_{j}} r_{B}^{Q}<\varepsilon .
$$

In the following we write $\mathcal{F}^{\varepsilon}:=\bigcup_{j=m_{\varepsilon}}^{\infty} \mathcal{F}_{j}$.

Covering a set with balls $B\left(f\left(x_{B}\right), r_{B}\right)$ may lead to an unoptimal covering and, thus, we distribute the "mass" of the ball to a sequence of balls of different scales. That is, we consider the following collection of weighted balls

$$
\mathcal{S}_{B}=\left\{\left(w_{k}, 2^{-k} B\left(f\left(x_{B}\right), r_{B}\right)\right): k \in \mathbf{N}, w_{k}=2^{k}\right\},
$$


with $B \in \mathcal{F}^{\varepsilon}$. This collection satisfies

$$
\sum_{B \in \mathcal{F}^{\varepsilon}} \sum_{\tilde{B} \in \mathcal{S}_{B}} w(\tilde{B}) r(\tilde{B})^{Q}=\sum_{B \in \mathcal{F}^{\varepsilon}} \sum_{i \in \mathbf{N}} w_{i} \frac{r_{B}^{Q}}{2^{i Q}} \leq C \sum_{B \in \mathcal{F}^{\varepsilon}} r_{B}^{Q}<C \varepsilon
$$

Here $w(B)$ denotes the weight associated to ball $B \in \mathcal{S}_{B}$.

Similarly we define $\mathcal{L}_{B}=\left\{\left(w_{k}, B\left(f\left(x_{B}\right), \alpha\left(2^{-k}\right)\right)\right): k \in \mathbf{N}, k+1 \geq \log _{2} r_{B}^{-1}, w_{k}=\right.$ $\lambda(k)\}$ with $B \in \mathcal{F}^{\varepsilon}$. Since the collection $\mathcal{S}_{B}$ already contains a ball with radius comparable to $\alpha\left(2^{-k}\right)$, which has larger weight than $B, \mathcal{L}_{B}$ is not entirely necessary. The purpose of introducing such notation is to make keeping track of balls easier. Using the fact that $\lambda(k)$ is increasing, analogously to (18) we obtain

$$
\begin{aligned}
\sum_{B \in \mathcal{F}^{\varepsilon}} \sum_{\tilde{B} \in \mathcal{L}_{B}} w(\tilde{B}) r(\tilde{B})^{Q} & =\sum_{B \in \mathcal{F}^{\varepsilon}} \sum_{\substack{i \in \mathbf{N} \\
i+1 \geq \log _{2} r_{B}^{-1}}} \lambda(i) \alpha\left(2^{-i}\right)^{Q} \\
& \leq \frac{1}{\lambda(0)^{Q-1}} \sum_{B \in \mathcal{F}^{\varepsilon}} \sum_{\substack{i \in \mathbf{N} \\
i+1 \geq \log _{2} r_{B}^{-1}}} 2^{-i Q} \\
& \leq \frac{C}{\lambda(0)^{Q-1}} \sum_{B \in \mathcal{F}^{\varepsilon}} r_{B}^{Q}<\frac{C}{\lambda(0)^{Q-1}} \varepsilon
\end{aligned}
$$

A suitable cover is constructed using elements of the collection $\mathcal{C}_{\varepsilon}=\bigcup_{B \in \mathcal{F}_{\varepsilon}} \mathcal{S}_{B} \cup$ $\mathcal{L}_{B}$. By (18) and (19) we have

$$
\sum_{B \in \mathcal{C}_{\varepsilon}} w(B) r(B)^{Q}=\sum_{B \in \mathcal{F}^{\varepsilon}} \sum_{\tilde{B} \in \mathcal{L}_{B}} w(\tilde{B}) r(\tilde{B})^{Q}+\sum_{B \in \mathcal{F}^{\varepsilon}} \sum_{\tilde{B} \in \mathcal{S}_{B}} w(\tilde{B}) r(\tilde{B})^{Q}<C \varepsilon .
$$

Let $x \in E$ and let $\left(B_{i}\right)_{i=1}^{\infty}$ be the approximating sequence converging to $x$ given in Definition 3.1 (A2). Assume that not all points in the sequence $\left(f\left(x_{B_{i}}\right)\right)_{i=m_{\varepsilon}}^{\infty}$ are equal to $f(x)$. Then we may define $j_{0}(x)$ to be the least integer such that at least one point in sequence $\left(f\left(x_{B_{i}}\right)\right)_{i=m_{\varepsilon}}^{\infty}$ is not in $B\left(f(x), 2^{-j_{0}(x)}\right)$. Let $E_{l}=\left\{y \in E: j_{0}(y) \leq l\right\}$. Notice that $j_{0}(x)$ depends also on $\varepsilon$. If $f\left(x_{B_{i}}\right)=f(x)$ for all $i=m_{\varepsilon}, m_{\varepsilon}+1, \ldots$, then we say that $x \in E_{\infty}$. Obviously $E=\cup_{l \in \mathbf{N} \cup\{\infty\}} E_{l}$. Notice that the cardinality of $E_{\infty}$ is at most the cardinality of $\mathcal{F}$ and, therefore, $E_{\infty}$ is countable.

To prove the claim we first consider only the set $E_{l_{0}}$ for some fixed $l_{0} \in \mathbf{N}$ and in the end use Lemma 2.3. In steps 1,2 and 3 below we construct the suitable weighted covering. Before that we will introduce some useful notation. Let $x \in E_{l_{0}}$ and let $\left(B_{i}\right)_{i=0}^{\infty}$ be an approximating sequence converging to $x$. By properties (A1), (A2) of Definition 3.1 and the uniform continuity of $f$ we see that $d\left(f\left(x_{B_{i}}\right), f(x)\right) \rightarrow 0$ as $i \rightarrow \infty$. For dyadic annuli we use following notation $A_{k}(f(x)):=B\left(f(x), 2^{-k}\right) \backslash$ $\bar{B}\left(f(x), 2^{-k-1}\right)$.

To find suitable balls for our covering we need to investigate how the sequence $\left(f\left(x_{B_{i}}\right)\right)_{i=0}^{\infty}$ approaches $f(E)$. Due to convergence this sequence crosses all annuli $A_{k}(f(x))$. Heuristically speaking, the set $P_{k}(f(x))$ we introduce below consists of balls which correspond to the last crossing of the annulus $A_{k}(f(x))$ by the sequence $\left(f\left(x_{B_{i}}\right)\right)_{i=0}^{\infty}$. In general, the sequence can cross the annulus several times but for our argument it suffices to look only at the last crossing. More precisely, for a given $k$ let $i_{k}=\max \left\{i \in \mathbf{N}: f\left(x_{B_{i}}\right) \in B\left(f(x), 2^{-k}\right)^{C}\right\}$ and $j_{k}=\min \left\{j \in \mathbf{N}: f\left(x_{B_{s+1}}\right) \in\right.$ $\bar{B}\left(f(x), 2^{-k-1}\right)$ and $\left.s \geq i_{k}\right\}$. If $j_{k}>i_{k}$ then we set $P_{k}(f(x))=\left\{B_{i_{k}+1}, B_{i_{k}+2}, \ldots, B_{j_{k}}\right\}$, and if $j_{k}=i_{k}$ we set $P_{k}(f(x))=\left\{B_{j_{k}+1}\right\}$. 
Step 1. We will show that in a significant number of annuli $A_{k}(x)$ there are not too many points of the sequence $\left(f\left(x_{B_{i}}\right)\right)_{i=m_{\varepsilon}}^{\infty}$. This implies that some of the corresponding radii $r_{B_{i}}$ must be large. Define

$$
\theta_{k}(x)= \begin{cases}1, & \text { if } \# P_{k}(x) \leq \frac{\tilde{c}}{4} \lambda(k), \\ 0, & \text { if } \# P_{k}(x)>\frac{\tilde{c}}{4} \lambda(k),\end{cases}
$$

where $\tilde{c}$ is a large constant, depending only on $\beta$ of (10), that will be fixed later. For every $k \in \mathbf{N}$ we have by our continuity assumption

$$
2^{-k-1} \leq\left|f(x)-f\left(x_{B_{j_{k}}}\right)\right| \leq \psi\left(C 2^{-j_{k}}\right),
$$

since, by (A1) and (A2), we have $\left|x-x_{B_{j_{k}}}\right| \leq C 2^{-j_{k}}$. This implies

$$
\psi^{-1}\left(2^{-k}\right) \leq C 2^{-j_{k}}
$$

We will show by a contradiction that there exists a number $l_{1} \in \mathbf{N}$, depending on $l_{0}$ and $\beta$ only, such that for every $l \geq l_{1}$

$$
\sum_{k=l_{0}}^{l} \theta_{k}(x) \geq \frac{l}{2} .
$$

Assume that there are arbitrarily large $l \in \mathbf{N}$ such that (22) does not hold. Recall that the number $j_{l}$ is the index of the last element in the last crossing of annulus $A_{l}(f(x))$. Thus, the number of elements in all $P_{k}\left(f(x)\right.$ with $\theta_{k}(f(x))=0, k=l_{0}, \ldots, l$, is smaller than $j_{l}$. Using this, (9) and (10) we may estimate the sum of $\# P_{k}(x)$ from below as follows:

$$
\begin{aligned}
j_{l} \geq & \sum_{\substack{k=l_{0} \\
\theta_{k}(x)=0}}^{l} \# P_{k}(f(x)) \geq \sum_{k=l_{0}}^{\lceil l / 2\rceil} \frac{\tilde{c}}{4} \lambda(k)=\frac{\tilde{c}}{4} \sum_{k=l_{0}}^{\lceil l / 2\rceil} \frac{2^{-k}\left(\psi^{-1}\right)^{\prime}\left(2^{-k}\right)}{\psi^{-1}\left(2^{-k}\right)} \\
& \geq \frac{\tilde{c}}{4} \int_{\left[2^{-\lceil l / 2\rceil}, 2^{\left.-l_{0}\right]}\right.} \frac{\left(\psi^{-1}\right)^{\prime}(t)}{\psi^{-1}(t)} d t \geq \frac{\tilde{c}}{4}\left(\log \frac{1}{\psi^{-1}\left(2^{-\lceil l / 2\rceil}\right)}-\log \frac{1}{\psi^{-1}\left(2^{-l_{0}}\right)}\right) \\
& \geq \frac{\tilde{c}}{4} \beta^{-1} \log \frac{1}{\psi^{-1}\left(2^{-l}\right)}-\frac{\tilde{c}}{4} \log \frac{1}{\psi^{-1}\left(2^{-l_{0}}\right)} .
\end{aligned}
$$

Combining (23) with (21) gives

$$
(\log 2)^{-1} \log \frac{1}{\psi^{-1}\left(2^{-l}\right)}+C\left(l_{0}\right) \geq \frac{\tilde{c}}{4} \beta^{-1} \log \frac{1}{\psi^{-1}\left(2^{-l}\right)} .
$$

If we choose $\tilde{c}>4(\beta \log 2)^{-1}$ then we get a contradiction for large $l$. Thus, we have found a constant $l_{1}$ as required. Moreover, notice that $l_{1}$ does not depend on the point under consideration, but on $l_{0}$ only.

Step 2. Consider now an annulus $A_{l}(f(x))$ for which $\theta_{l}(f(x))=1$. We show that there exists a set of balls $\left\{B\left(f\left(x_{B_{i}}\right), r_{B_{i}}\right): i=1, \ldots m\right\} \subset \mathcal{C}_{\varepsilon}$, satisfying

$$
r_{B_{i}} \geq C_{1} \alpha\left(2^{-l}\right)
$$

for all $i$ and

$$
\sum_{i=1}^{m} w_{i} \geq C_{2} \lambda(l)
$$

Here $C_{1}$ and $C_{2}$ are positive constants independent of $x$ and $l$. 
By triangle inequality and 3.2, the sum of numbers $2 r_{B}$ related to a crossing of $A_{l}(f(x))$ must be larger than the width of the annulus. That is, we have the following inequality

$$
\sum_{B \in P_{l}(f(x))} 2 r_{B} \geq 2^{-l-1}
$$

The upper bound on the cardinality of $P_{l}(f(x))$ implies that not all of the radii can be small, in fact, it is not hard to see that

$$
\sum_{\substack{B \in P_{l}(f(x)) \\ r_{B} \geq \alpha\left(2^{-l}\right) / 2 \tilde{c}}} 2 r_{B} \geq 2^{-l-2} .
$$

Assume that $\left\{f\left(x_{B}\right): B \in P_{l}(f(x))\right\}$ contains points in $A_{l}(f(x))$. Given a ball $B \in P_{l}(f(x))$ such that $f\left(x_{B}\right) \in A_{l}(f(x))$ and $r_{B} \geq \alpha\left(2^{-l}\right) / 2 \tilde{c}$, we find a number $n_{B} \in \mathbf{N}$ for which

$$
\frac{\alpha\left(2^{-l}\right)}{4 \tilde{c}} \leq \frac{r_{B}}{2^{n_{B}}} \leq \frac{\alpha\left(2^{-l}\right)}{2 \tilde{c}} .
$$

For such $B \in P_{l}(f(x))$, we denote $\left(w_{\tilde{B}}, \tilde{B}\right):=\left(2^{n_{B}}, B\left(f\left(x_{B}\right), \frac{r_{B}}{2^{n} B}\right)\right)$. Notice that $\left(w_{\tilde{B}}, \tilde{B}\right) \in \mathcal{S}_{B}$. By definition we have $r(\tilde{B}) \geq \frac{\alpha\left(2^{-l}\right)}{4 \tilde{c}}$. Moreover, we can estimate the sum of weights

$$
2^{-l-2} \leq \sum_{\substack{B \in P_{l}(f(x)) \\ r_{B} \geq \alpha\left(2^{-l}\right) / 2 \tilde{c}}} 2 r_{B} \leq \frac{\alpha\left(2^{-l}\right)}{\tilde{c}} \sum_{\substack{B \in P_{l}(f(x)) \\ r_{B} \geq \alpha\left(2^{-l}\right) / 2 \tilde{c}}} 2^{n_{B}} \leq \frac{\alpha\left(2^{-l}\right)}{\tilde{c}} \sum_{\substack{B \in P_{l}(f(x)) \\ r_{B} \geq \alpha\left(2^{-l}\right) / 2 \tilde{c}}} w_{\tilde{B}} .
$$

This shows that such balls have the required properties, and we denote the set of such balls by $\mathcal{R}_{l}(f(x))$.

On the other hand, if $\left\{f\left(x_{B}\right): B \in P_{l}(f(x))\right\}$ contains no points of the set $A_{l}(f(x))$ then again by the definition of $P_{l}(f(x))$ and 3.2 we have that $r_{B} \geq 2^{-l-1}$. In this case the ball $\left(\lambda(l), B\left(f\left(x_{B}\right), \alpha\left(2^{-l}\right)\right)\right) \in \mathcal{L}_{B}$ satisfies the requirements and we denote by $\mathcal{R}_{l}(f(x))$ the set containing this ball.

Step 3. The balls we have chosen might be too small to cover the set $f\left(E_{l_{0}}\right)$ and we may have to enlarge them. For technical reasons we enlarge them by the same factor. This, on the other hand, might make some balls too large, and we have to compensate this by scaling the balls and changing the weight once more.

Let $l \geq l_{1}$. Take any $B \in \mathcal{C}_{\varepsilon}$. We define a ball $\tilde{B}$ by scaling $B$ as follows. If there is an integer $k_{B}$, such that $l_{0} \leq k_{B} \leq l$ and $\alpha\left(2^{-k_{B}}\right) / 4 \tilde{c} \leq r(B)<\alpha\left(2^{-k_{B}+1}\right) / 4 \tilde{c}$, we define $\tilde{B}=\frac{\lambda\left(k_{B}\right)}{\lambda(l)} B$ and the weight $w_{\tilde{B}}=\left(\frac{\lambda(l)}{\lambda\left(k_{B}\right)}\right)^{Q} w_{B}$. If $r(B) \geq \alpha\left(2^{-l_{0}+1}\right) / 4 \tilde{c}$, then we define $\tilde{B}=\frac{\lambda\left(l_{0}\right)}{\lambda(l)} B$ and the weight $w_{\tilde{B}}=\left(\frac{\lambda(l)}{\lambda\left(l_{0}\right)}\right)^{Q} w_{B}$. None of the balls with $r(B)<\alpha\left(2^{-l}\right) / 4 \tilde{c}$ are needed in the construction of the covering and they are removed. The set of balls obtained from $\mathcal{C}_{\varepsilon}$ after these modifications and removals is called $\mathcal{C}_{\varepsilon, l}$.

Next, we show that we can cover the set $f\left(E_{l_{0}}\right)$ with suitable balls and obtain a quantitative lower bound for the overlap of the cover. Towards this end, fix a point $f(x) \in f\left(E_{l_{0}}\right)$. Choose $k \in\left\{l_{0}, \ldots, l\right\}$ such that $\theta_{k}(f(x))=1$. Let $B_{i} \in \mathcal{R}_{k}(f(x))$ be the balls chosen in step 2. For these balls we have $r\left(B_{i}\right) \geq \frac{\alpha\left(2^{-k}\right)}{4 \tilde{c}}$ and, therefore, $k_{B_{i}} \leq k$. In the case $r\left(B_{i}\right) \geq \frac{\alpha\left(2^{-l_{0}}\right)}{4 \tilde{c}}$, we set $k_{B_{i}}=l_{0}$ in the following calculations. 
We have the estimate

$$
\sum_{B_{i} \in \mathcal{R}_{k}(f(x))} w_{\tilde{B}_{i}}=\sum_{B_{i} \in \mathcal{R}_{k}(f(x))}\left(\frac{\lambda(l)}{\lambda\left(k_{i}\right)}\right)^{Q} w_{B_{i}} \geq\left(\frac{\lambda(l)}{\lambda(k)}\right)^{Q} \sum_{B_{i} \in \mathcal{R}_{k}(f(x))} w_{B_{i}} \geq \frac{C_{2} \lambda(l)^{Q}}{\lambda(k)^{Q-1}} .
$$

Also, $r\left(\tilde{B}_{i}\right) \geq \frac{2^{-k}}{4 \tilde{c} \lambda(l)}$. Since the center of the ball is in $B\left(f(x), 2^{-k}\right)$, the ball $4 \tilde{c} \lambda(l) \tilde{B}_{i}$ contains the point $f(x)$.

We now estimate the total overlap. Let $y \in f\left(E_{l_{0}}\right)$. By $(29)$ we have the estimate

$$
\sum_{B \in \mathcal{C}_{\varepsilon, l}} w_{B} \chi_{4 \tilde{c} \lambda(l) B}(y) \geq \sum_{\substack{k=l_{0}, \ldots, l \\ \theta_{k}(y)=1}} C_{2} \frac{\lambda(l)^{Q}}{\lambda(k)^{Q-1}} \geq C_{2} \frac{\lambda(l)^{Q}}{4} \sum_{k=l_{1}}^{l} \lambda(k)^{1-Q} .
$$

We use the notation $G(l):=\sum_{k=l_{1}}^{l} \lambda(k)^{1-Q}$.

Step 4. So far we have shown that $\left(\frac{4 w_{B}}{C_{2} \lambda(l)^{Q} G(l)}, 4 \tilde{c} \lambda(l) B\right)_{B \in \mathcal{C}_{\varepsilon, l}}$ is a weighted cover of the set $f\left(E_{l_{0}}\right)$. We note that $4 \tilde{c} \lambda(l) r(B) \geq 2^{-l}$, for every ball $B$ in $\mathcal{C}_{\varepsilon, l}$.

Before estimating the Hausdorff content we have to make two observations. First, by our integrability condition (16) we have

$$
G_{l}=\sum_{j=l_{1}}^{l} \frac{1}{\lambda(j)^{Q-1}}=\sum_{j=l_{1}}^{l}\left(\frac{\psi^{-1}\left(2^{-j}\right)}{2^{-j}\left(\psi^{-1}\right)^{\prime}\left(2^{-j}\right)}\right)^{Q-1} \geq \int_{2^{-l}}^{2^{-l_{1}}}\left(\frac{\psi^{-1}(t)}{\left(\psi^{-1}\right)^{\prime}(t)}\right)^{Q-1} \frac{d t}{t^{Q}}
$$

and, thus, $G_{l} \rightarrow \infty$ as $l \rightarrow \infty$. This implies that

$$
\eta\left(2^{-l}\right) \leq \sum_{k=1}^{l_{1}-1} \frac{1}{\lambda(k)^{Q-1}}+G_{l} \leq 2 G(l),
$$

for large enough $l$. Recall that $\eta\left(2^{-l}\right)$ is factor of $2^{-l n}$ in our gauge function. Second thing is that $(20)$ holds for family $\mathcal{C}_{\varepsilon, l}$. Now we have all required tools to estimate the weighted Hausdorff content:

$$
\begin{aligned}
\mathcal{H}_{w, \infty}^{h}\left(f\left(E_{l_{0}}\right)\right) & \leq \frac{4}{C_{2} \lambda(l)^{Q} G(l)} \sum_{B \in \mathcal{C}_{\varepsilon, l}} w_{B}(\operatorname{diam} 4 \tilde{c} \lambda(l) r(B))^{Q} \eta\left(2^{-l}\right) \\
& \leq C \sum_{B \in \mathcal{C}_{\varepsilon, l}} w_{B} r(B)^{Q} \leq C \varepsilon
\end{aligned}
$$

Notice that above the constant $C$ is independent of $l_{0}$. Now, Lemma 2.4 implies $\mathcal{H}_{\infty}^{h}\left(f\left(E_{l_{0}}\right)\right) \leq C \varepsilon$ and by Lemma 2.3 we obtain $\mathcal{H}_{\infty}^{h}(f(E)) \leq C \varepsilon$. Since $\varepsilon$ is arbitrary, we have $\mathcal{H}_{\infty}^{h}(f(E))=0$, which implies $\mathcal{H}^{h}(f(E))=0$.

Remark. The integrability condition (3) is needed only in the last step of the proof. The proof works also without this condition, if we redefine $\eta$. It is enough assume that there exists a constant $C$, independent of $l_{1}$, such that

$$
\eta\left(2^{-l}\right) \leq C G(l)
$$

for large $l$.

It is important to notice that if (3) is not satisfied we might have $\mathcal{H}^{Q}(f(E))>0$. The reason for failure of the proof above is that if $l_{0}$ and $l_{1}$ are large $G_{l}$ is small and the ratio $\frac{\varepsilon}{G_{l}}$ might not be bounded. Remember that $\varepsilon$ was fixed before partitioning the set $f(E)$ into $f\left(E_{l_{0}}\right)$ and now $\eta\left(2^{-k}\right)=1$. This coincides with [7, Theorem C.1] and $[8$, Theorem 1.1]. 


\section{The proof of Theorem 1.1}

Before the proof of Theorem 1.1 in Lemma 4.2 we will show that if $E$ is a porous set in a $Q$-Ahlfors regular space then there is an approximating family of $E$. We use the following metric space analogue of the usual Whitney decomposition. Let $X$ be a $Q$-Ahlfors regular metric measure space and $1>\gamma>0$. Let $E \subset X$ be closed. For all points $x \in X$ we denote $d(x):=d(x, E)$. The $\gamma$-Whitney cover $\mathcal{W}_{\gamma}$ of $X \backslash E$ is a maximal collection of balls $B_{i}:=B\left(z_{i}, \gamma \frac{d\left(z_{i}\right)}{10}\right), i \in I$ such that the balls $\frac{1}{5} B_{i}$ are disjoint. Some other well-known properties of $\mathcal{W}_{\gamma}$ are listed in the following lemma. The last property follows from $Q$-regularity, but holds in more general settings too, for example when the measure $\mu$ is doubling.

Lemma 4.1. Let $X$ be a $Q$-Ahlfors regular metric measure space and let $E \subset X$ be a closed set. The $\gamma$-Whitney covering of $X \backslash E$ exists and has the following properties:

(1)

$$
\bigcup_{i \in I} B_{i}=X \backslash E
$$

(2) for all $i \in I$,

$$
5 B_{i} \subset X \backslash E,
$$

(3) if $x \in B\left(z, \gamma \frac{d(z)}{10}\right) \in \mathcal{W}_{\gamma}$ then $\frac{9}{10} d(z) \leq d(x) \leq \frac{11}{10} d(z)$,

(4) for all $x \in X$,

$$
\sum_{i \in I} \chi_{5 B_{i}}(x) \leq N
$$

Lemma 4.2. Let $X$ be a $Q$-Ahlfors regular metric measure space Let $E \subset X$ be a closed $\alpha$-porous. Then there is an approximating family $\left(\mathcal{F}_{n}\right)_{n \in \mathbf{N}}$ of $E$ and $N \in \mathbf{N}$ such that

$$
\sum_{n \in \mathbf{N}} \sum_{B \in \mathcal{F}_{n}} \chi_{B} \leq N
$$

Proof. Let $\gamma=5 \alpha$ and let $\mathcal{W}_{\gamma}$ be a $\gamma$-Whitney decomposition of $X \backslash E$. Let $x \in E$ be an arbitrary point and $r>0$ arbitrary small radius. Let $B(z, \alpha r) \subset B(x, r) \backslash E$ be a ball given by the porosity condition. By Lemma 4.1 4.1 there exists a ball $B:=B\left(y, \frac{\lambda d(y)}{10}\right) \in \mathcal{W}_{\gamma}$ which contains point $z$, and which is contained in $B(z, \alpha r)$. Our choice of $\gamma$ and Lemma 4.14 .1 imply that

$$
\frac{\gamma^{2}}{100} r<r(B) \leq \frac{\gamma}{10} r
$$

We define $\mathcal{F}_{n}=\left\{B \in \mathcal{W}_{\gamma}: \frac{\gamma^{2}}{100} 2^{-n}<r(B) \leq \frac{\gamma}{10} 2^{-n}\right\}$. Notice that while a certain ball can belong to $\mathcal{F}_{n}$ for different numbers $n$, the number of such $n$ is bounded, with a bound depending on $\gamma$ only. This fact and Lemma 4.14 .1 imply (33).

For a given $x \in E$, we obtain the approximating sequence by choosing $B_{n}$ to be the ball $B \in \mathcal{W}_{\gamma}$ chosen in (34). This choice has the desired properties.

Proof of Theorem 1.1. Let $\mathcal{W}_{\gamma}$ be a Whitney decomposition of $X \backslash E$ and $\left(\mathcal{F}_{n}\right)_{n \geq 0}$ the approximating family given by Lemma 4.2. Given an arbitrary ball $B \in \mathcal{W}_{\gamma}$ there exists a point $x_{B} \in B$ such that

$$
\left(f_{B} g^{Q} d \mu\right)^{\frac{1}{Q}} \geq g\left(x_{B}\right)
$$


and $x_{B} \in X \backslash A$, where $A$ is the exceptional set in Definition 2.1.

For a given $B \in \mathcal{F}_{j}$, we define

$$
r_{B}=\max _{B \sim \tilde{B}}\left\{d\left(f\left(x_{B}\right), f\left(x_{\tilde{B}}\right)\right)\right\}
$$

where $B \sim \tilde{B}$ means that $\tilde{B} \in \mathcal{F}_{k}, k=j-1, j, j+1$, and $d(B, \tilde{B}) \leq 2^{2-j}$. By Ahlfors regularity the number of balls $\tilde{B}$ with $\tilde{B} \sim B$ is bounded from above by a constant $M$. Notice that 3.2 is satisfied since we have $B_{i} \sim B_{i+1}$ and $B_{i} \sim B_{i-1}$ for all balls in the approximating sequence of a point $x \in E$. This follows from the proof of Lemma 4.2 , since all three balls $B_{i}, B_{i+1}$, and $B_{i-1}$ are chosen so that they are contained in the ball $B\left(x, 2^{-i+1}\right)$.

For any balls $B, B^{\prime} \in \mathcal{F}$ with $B \sim B^{\prime}$ we know that numbers $r(B), r\left(B^{\prime}\right)$ and $d\left(x_{B}, x_{B^{\prime}}\right)$ are all comparable with constant independent of $B$ and $B^{\prime}$. This, Ahlfors regularity of measure $\mu$, and the definition Hajłasz gradient imply

$d\left(f\left(x_{B}\right), f\left(x_{B^{\prime}}\right)\right)^{Q} \leq C d\left(x_{B}, x_{B^{\prime}}\right)^{Q}\left(f_{B} g^{Q} d \mu+f_{B^{\prime}} g^{Q} d \mu\right) \leq C \int_{B} g^{Q} d \mu+C \int_{B^{\prime}} g^{Q} d \mu$.

This implies by Lemma 4.14 .1 that

$$
r_{B}^{Q} \leq C \sum_{\substack{B^{\prime} \\ B \sim B^{\prime}}}\left(\int_{B} g^{Q} d \mu+\int_{B^{\prime}} g^{Q} d \mu\right) \leq C M \int_{\cup_{B \sim B^{\prime}} B^{\prime}} g^{Q} d \mu .
$$

Summing the previous inequality over all $B \in \mathcal{F}$ we obtain by 4.1

$$
\sum_{B \in \mathcal{F}} r_{B}^{Q} \leq C M^{2} \int_{X \backslash E} g^{Q} d \mu<\infty
$$

This means that the pointed approximating family $\left\{\left(x_{B}, B\right): B \in \mathcal{F}\right\}$ and sequence $\left(r_{B}\right)_{B \in \mathcal{F}}$ satisfy the assumptions in Proposition 3.2, which implies the claim.

\section{Example}

In this section we show the sharpness of the Theorem 1.1. Our example is a modification of the example given in [9]. Assume $Q>1$ is a real number and $n \geq Q$ an integer. Given an allowable modulus of continuity $\psi$ satisfying (3) with $\psi(t) \geq t^{a}$, with $a<\frac{1}{n}$, and any number $\beta>1$ we construct a mapping from a $Q$-Ahlfors regular space $X$ to $\mathbf{R}^{n}$ with $L^{Q}$-Hajłasz gradient and modulus of continuity $C \psi$, which maps a porous set to a set with positive $\mathcal{H}^{h}$-measure. Here $h$ is an increasing function satisfying

$$
h\left(2^{-j}\right)=2^{-j Q}\left(\sum_{k=1}^{j} \frac{1}{\lambda(k)^{Q-1}}\right)^{\beta} .
$$

We choose our space $X$ to be a compact $Q$-regular metric measure space, which is geodesic and satisfies a weak (1,1)-Poincaré inequality and has a piece of a geodesic which is porous. Examples of suitable spaces are Laakso spaces [11] for non-integer $Q$ and for integer $Q$ the closed unit ball of $\mathbf{R}^{Q}$ with Lebesque measure and Euclidean metric. We also denote $\psi^{-1}$ by $u$ and the function $\lambda$ is as in proof of Proposition 3.2.

5.1. Domain Cantor set. Let $I \subset X$ be a segment of a geodesic. We define numbers $R_{k}:=2^{-n} u\left(2^{-k}\right)$ and $r_{k}:=u\left(2^{-k-1}\right), \forall k \in \mathbf{N}$. Let $k_{0} \in \mathbf{N}$ be a large constant, which will be fixed later, such that $2 r_{k_{0}}$ is smaller than the length of $I$. We choose a subinterval $I_{k_{0}} \subset I$ of length $2 r_{k_{0}}$. 
Notice that by (9) we have

$$
\log \frac{R_{k}}{r_{k}}=\log \frac{1}{u\left(2^{-k-1}\right)}-\log \frac{1}{u\left(2^{-k}\right)}-n \log 2 \geq \log 2 \frac{u^{\prime}\left(2^{-k}\right)}{u\left(2^{-k}\right)} 2^{-k}-n \log 2 .
$$

Now if the last expression were non-positive for all large $k$, we would obtain from Grönwall's inequality that $u\left(2^{-k}\right) \geq 2^{-n k}$. This contradicts our assumption $\psi(t) \geq t^{a}$, with $a<1 / n$. In fact, we obtain following inequalities

$$
\log \frac{R_{k}}{r_{k}} \geq \log 2\left(\frac{u^{\prime}\left(2^{-k}\right)}{u\left(2^{-k}\right)} 2^{-k}-n\right) \geq C(a)>0,
$$

which are valid for all large integers $k$. This inequality is needed to guarantee that $R_{k}>r_{k}$ in the following construction.

We split $I_{k_{0}}$ to $2^{n}$ subintervals of equal length with disjoint interiors and denote their center points as $a_{k_{0}, i}, i=1,2, \ldots, 2^{n}$. These subintervals are denoted by $I_{k_{0}+1, i}^{\prime}$, and their lengths are equal to $2 R_{k_{0}+1}$. By $I_{k_{0}+1, i}, i=1,2, \ldots, 2^{n}$, we denote the intervals of length $2 r_{k_{0}+1}$ and centers $a_{k_{0}, i}$. We continue inductively. Assume that for some $l>k_{0}$ we have defined intervals $I_{l, i}, I_{l, i}^{\prime}$ with centers $a_{l, i}, i=1,2, \ldots, 2^{n\left(l-k_{0}\right)}$ and of lengths $2 r_{l}$ and $2 R_{l}$, respectively. We split each $I_{l, i}$ to $2^{n}$ disjoint parts of length $2 R_{l+1}$ and denote these by $I_{l+1, i}^{\prime}$. Let $a_{l, i}, i=1,2, \ldots, 2^{n\left(l+1-k_{0}\right)}$ be the centers of these intervals and define $I_{l+1, i}$ to be intervals of lengths $2 r_{l+1}$ with centers $a_{l+1, i}$.

In Sections 5.4 and 5.5 we will use the following notations $B_{j, i}=B\left(a_{j, i}, r_{j}\right)$, $B_{j, i}^{\prime}=B\left(a_{j, i}, R_{j}\right)$, and $A_{j, i}:=\overline{B_{j, i}^{\prime}} \backslash B_{j, i}$. We will construct a mapping which maps the set

$$
C_{d}=\bigcap_{j=k_{0}+1}^{\infty} \bigcup_{i=1}^{2^{n\left(j-k_{0}\right)}} I_{j, i}
$$

to a set of positive $\mathcal{H}^{h}$-measure.

5.2. Target Cantor set. Remember that $n \geq Q$ is some integer fixed we fixed in the beginning of Section 5 . We will construct a Cantor set in $\mathbf{R}^{n}$ of correct size. Define $d_{j}=2^{-j \frac{n}{Q}}\left(\sum_{k=1}^{j} \frac{1}{\lambda(k)^{Q-1}}\right)^{-\beta / Q}$. Notice that $0<d_{k}$ and

$$
2 \leq \frac{d_{k-1}}{d_{k}}<2^{\frac{n+\beta}{Q}}
$$

for all $k$.

Let us start with a cube $K_{k_{0}}$ of $\mathbf{R}^{n}$, that is the cube with center 0 and edge length $d_{k_{0}}$. Here $k_{0}$ is the same number as in the Section 5.1. Define $K_{k_{0}+1, i}, i=1,2, \ldots, 2^{n}$, to be the cubes of edge length $d_{k_{0}+1}$ located at the corners of $K_{k_{0}}$. Notice, that by our assumption on the numbers $d_{j}$ the cubes are disjoint. We continue inductively. Assume that we are given a collection of disjoint cubes $K_{j, i}$, with integers $j \geq k_{0}$ and $i=1,2, \ldots, 2^{n\left(j-k_{0}\right)}$. From each corner of each of these cubes we choose a cube with edge length equal to $d_{j+1}$. We denote these cubes by $K_{j+1, i}$, where $i=$ $1,2, \ldots, 2^{\left(j+1-k_{0}\right) n}$. These cubes are disjoint by the choice of the numbers $d_{k}$ and the fact that $K_{j, i}$ are disjoint.

We define

$$
C_{t}=\bigcap_{j=k_{0}+1}^{\infty} \bigcup_{i=1}^{2^{n\left(j-k_{0}\right)}} K_{j, i} .
$$


The set $C_{t}$ will be contained in the image of the map we will define in the next section.

We will now estimate the dimension of this set. According to Lemma 2.5, it suffices to find a finite nonzero Radon measure $\mu$ with support in $C_{t}$, such that for every $B(x, r)$, we have $\mu(B(x, r)) \leq c h(r)$. We construct such a measure as a limit of sequence of measures. We define measures $\left\{\mu_{m}\right\}_{m=k_{0}+1}^{\infty}$ by defining $\mu_{m}$ to be the measure of total mass 1 , supported on $\bigcap_{j=k_{0}+1}^{m} \bigcup_{i=1}^{2^{n\left(j-k_{0}\right)}} K_{j, i}$, absolutely continuous with respect to Lebesgue measure and having uniform density. Since this sequence is bounded, by compactness, we find a weakly convergent subsequence, which is, for simplicity, denoted by $\left\{\mu_{m}\right\}_{m=k_{0}}^{\infty}$ and the limit measure by $\mu$ (see [12, Theorem 1.23]). It is obvious that $\mu$ is supported in $C_{t}$.

Now, let $x \in C_{t}, r>0$ suitably small number and consider the ball $B(x, r)$. Let $\theta$ be a continuous real-valued function with support in $B(x, 2 r)$ and $\theta \equiv 1$ in $B(x, r)$. By the definition of numbers $d_{j}$ (see (42)) we find an integer $j$ such that

$$
2^{-k} \leq r \leq 2^{-k+1} \text { and } 2^{-k} \leq d_{j} \leq 2^{\frac{n+\beta}{Q}} 2^{-k}
$$

Let us assume that the number cubes of generation $j$ the ball $B(x, 2 r)$ intersects is $M$. Since diameter of all of these cubes is $\sqrt{n} d_{j}$, we see that all these cubes are contained in the ball $B\left(x, 2 r+\sqrt{n} d_{j}\right)$. Comparing the volumes of cubes and the ball we obtain an upper bound for $M$ independent of $j$ and $r$.

By the construction of the measures $\mu_{m}$, we have the following estimate

$$
\mu(B(x, r)) \leq \int \theta d \mu=\lim _{m \rightarrow \infty} \int \theta d \mu_{m} \leq \lim _{m \rightarrow \infty} \mu_{m}\left(\bigcup_{l=1}^{M} K_{j, i_{l}}\right)=C 2^{-n j}
$$

On the other hand we have for large $j$ and $k$

$$
\begin{aligned}
h(r) & \geq h\left(2^{-k}\right) \geq C 2^{-k Q}\left(\sum_{l=1}^{k} \frac{1}{\lambda(l)^{Q-1}}\right)^{\beta} \\
& \geq C 2^{-j n}\left(\sum_{l=1}^{j} \frac{1}{\lambda(l)^{Q-1}}\right)^{-\beta}\left(\sum_{l=1}^{k} \frac{1}{\lambda(l)^{Q-1}}\right)^{\beta} \geq C 2^{-j n} .
\end{aligned}
$$

The sums above cancel since our choice of $j$ implies $j \leq k$.

Now the desired dimension estimate follows from Frostman lemma by inequalities (45) and (46).

5.3. Mapping. The idea is to construct a continuous map $f$ which maps the geodesic segment $I_{k_{0}}$ on a curve which contains the Cantor set $C_{t}$. This is achieved by showing that the centers of all cubes $K_{j, i}$ are in $f\left(I_{k_{0}}\right)$, then by the compactness of $I_{k_{0}}$ and continuity of $f$ we know that $C_{t} \subset f\left(I_{k_{0}}\right)$. The map $f$ is obtained as a sum of maps $\varphi_{j, i}$. These maps map certain annuli $A_{j, i}$ defined in Section 5.1 to lines, which connect center of cube of generation $j$ to cube generation $j+1$.

To define $\varphi_{j, i}$ we first define auxiliary functions $\varphi_{j}$. We use logarithms in order to achieve the required integrability of Hajłasz gradient of $f$. Let $R_{j}$ and $r_{j}$ be as 
above. Let $\varphi_{j}:[0, \infty) \rightarrow[0,1]$ be the map given by

$$
\varphi_{j}(x)= \begin{cases}\frac{\log \frac{R_{j}}{R_{j}}}{\log \frac{R_{j}}{r_{j}}}, & \text { if } r_{j} \leq x \leq R_{j}, \\ 0, & \text { if } x>R_{j}, \\ 1, & \text { if } x<r_{j} .\end{cases}
$$

Let $v:\left\{1,2, \ldots, 2^{n}\right\} \rightarrow\left\{\left(x_{1}, x_{2}, \ldots x_{n}\right) \in \mathbf{R}^{n}: x_{i}= \pm 1 \forall i\right\}$ be a bijection. In the following we use the notation $v_{i}=v(i)$. Moreover, we define $v_{i}=v_{i-2^{n}}$ for $i>2^{n}$.

We now define a mapping from $X$ to $\mathbf{R}^{n}$ by setting

$$
\varphi_{j, i}(x)=d_{j} \frac{v_{i}}{2}\left(1-\frac{d_{j+1}}{d_{j}}\right) \varphi_{j}\left(d\left(x, a_{j, i}\right)\right) .
$$

This map is supported on $B_{j, i}^{\prime}$ and its local Lipschitz constant is supported on $A_{j, i}=$ $B_{j, i}^{\prime} \backslash B_{j, i}$. Finally, summing over both indices we obtain a map $f: X \rightarrow \mathbf{R}^{n}$, given by

$$
f(x)=\sum_{j=k_{0}+1}^{\infty} \sum_{i=1}^{2^{\left(j-k_{0}\right) n}} \varphi_{j, i}(x) .
$$

This mapping is continuous as a uniform limit of continuous mappings, since for every $j$

$$
\left|\sum_{i=1}^{2^{\left(j-k_{0}\right) n}} \varphi_{j, i}(x)\right| \leq \frac{\sqrt{n}}{2} d_{j} \leq \frac{\sqrt{n}}{2} 2^{-j} .
$$

By the construction, the image of the segment $I_{k_{0}}$ contains the centers of all cubes $K_{j, i}$ in the construction of $C_{t}$. By the continuity of $f$ and the compactness $I_{k_{0}}$ the set $f\left(I_{k_{0}}\right)$ is also compact and, therefore, it must contain $C_{t}$.

5.4. Integrability of the derivative. Now we will show that the mapping $f$ defined in the previous section satisfies the required integrability condition. First, notice that since our space supports $(1,1)$-Poincaré inequality it is enough to show that $f$ has a $Q$-integrable upper gradient (see [5, Corollary 9.1.10]). A good candidate for an upper gradient is the local Lipschitz constant (see [5, Lemma 5.2.8]). Our functions $\varphi_{j}$ are locally Lipschitz with constants

$$
\operatorname{lip}\left(\varphi_{j}\right)(x)= \begin{cases}\left(\log \frac{R_{j}}{r_{j}}\right)^{-1} \frac{1}{|x|}, & \text { if } r_{j} \leq x \leq R_{j} \\ 0, & \text { otherwise. }\end{cases}
$$

For the calculations we need the following elementary lemma.

Lemma 5.1. Let $x$ be a point in a $Q$-Ahlfors regular metric measure space $X$. Given two real numbers $r$ and $R$, such that $0<r<R<r_{0}$, we have

$$
\int_{B(x, R) \backslash B(x, r)} \frac{1}{d(x, y)^{Q}} d \mu(y) \leq C \log \frac{R}{r} .
$$

The constant $C$ depends only on the constants of Ahlfors regularity and $r_{0}$ is the radius in the definition of Ahlfors regularity. 
Proof. A straightforward calculation gives

$$
\begin{aligned}
\int_{B(x, R) \backslash B(x, r)} \frac{1}{d(x, y)^{Q}} d \mu(y) & \leq \sum_{i=0}^{\left\lfloor\log _{2} \frac{R}{r}\right\rfloor} \int_{B\left(x, 2^{i+1} r\right) \backslash B\left(x, 2^{i} r\right)} \frac{1}{d(x, y)^{Q}} d \mu(y) \\
& \leq \sum_{i=0}^{\left\lfloor\log _{2} \frac{R}{r}\right\rfloor} \int_{B\left(x, 2^{i+1} r\right) \backslash B\left(x, 2^{i} r\right)} 2^{-i Q} r^{-Q} d \mu(y) \\
& \leq \sum_{i=0}^{\left\lfloor\log _{2} \frac{R}{r}\right\rfloor} C 2^{Q} \leq C 2^{Q} \log _{2} \frac{R}{r}=C 2^{Q} \log \frac{R}{r}
\end{aligned}
$$

Finally, we show the required integrability for the derivative. Using Lemma 5.1 we obtain

$$
\begin{aligned}
\int_{X}|D f|^{Q} d \mu \leq \sum_{j=k_{0}+1}^{\infty} \sum_{i=1}^{2^{j n}} \int_{A_{j, i}}|D f|^{Q} d \mu \\
\leq \sum_{j=k_{0}+1}^{\infty} \sum_{i=1}^{2^{j n}} d_{j}^{Q} \log \left(\frac{R_{j}}{r_{j}}\right)^{-Q} \int_{A_{j, i}} \frac{1}{d\left(x, a_{j, i}\right)^{Q}} d \mu \\
\leq \sum_{j=k_{0}+1}^{\infty} \sum_{i=1}^{2^{j n}} 2^{-j n}\left(\sum_{k=1}^{j} \frac{1}{\lambda(k)^{Q-1}}\right)^{-\beta} \log \left(\frac{R_{j}}{r_{j}}\right)^{1-Q} \\
\leq \sum_{j=k_{0}+1}^{\infty}\left(\sum_{k=1}^{j} \frac{1}{\lambda(k)^{Q-1}}\right)^{-\beta} \log \left(\frac{R_{j}}{r_{j}}\right)^{1-Q} \\
=\sum_{j=k_{0}+1}^{\infty}\left(\sum_{k=1}^{j}\left(\frac{u^{\prime}\left(2^{-k}\right)}{u\left(2^{-k}\right)} 2^{-k}\right)^{1-Q}\right)^{-\beta} \log \left(\frac{R_{j}}{r_{j}}\right)^{1-Q} .
\end{aligned}
$$

If $\frac{u^{\prime}\left(2^{-k}\right)}{u\left(2^{-k}\right)} 2^{-k}$ is bounded from above, then it is easy to show with the help of $(40)$ that the sum above converges. On the other hand, if this expression is not bounded then (40) implies that

$$
\log \frac{R_{k}}{r_{k}} \geq \frac{1}{2} \frac{u^{\prime}\left(2^{-k}\right)}{u\left(2^{-k}\right)} 2^{-k},
$$

for large $k$. Together with (53) this gives

$$
\int_{X}|D f|^{Q} d \mu \leq C+C \sum_{j=N+2}^{\infty}\left(\sum_{k=N}^{j}\left(\frac{u^{\prime}\left(2^{-k}\right)}{u\left(2^{-k}\right)} 2^{-k}\right)^{1-Q}\right)^{-\beta}\left(\frac{u^{\prime}\left(2^{-k}\right)}{u\left(2^{-k}\right)} 2^{-k}\right)^{1-Q}
$$

Now we are done since, for a positive sequence $\left\{a_{k}\right\}_{k=1}^{\infty}$ with infinite sum, the mean value theorem implies that

$$
\left(\sum_{k=N}^{j} a_{k}\right)^{1-\beta}-\left(\sum_{k=N}^{j-1} a_{k}\right)^{1-\beta} \leq(1-\beta) a_{j}\left(\sum_{k=N}^{j} a_{k}\right)^{-\beta}
$$


which implies

$$
\begin{aligned}
\sum_{j=N+2}^{\infty} a_{j}\left(\sum_{k=N}^{j-1} a_{k}\right)^{-\beta} & \leq(\beta-1)^{-1} \sum_{j=N+2}^{\infty}\left(\left(\sum_{k=N}^{j-2} a_{k}\right)^{1-\beta}-\left(\sum_{k=N}^{j-1} a_{k}\right)^{1-\beta}\right) \\
& \leq(\beta-1)^{-1} a_{N}^{1-\beta}-(\beta-1)^{-1} \lim _{m \rightarrow \infty}\left(\sum_{k=N}^{m} a_{k}\right)^{1-\beta} \\
& =(\beta-1)^{-1} a_{N}^{1-\beta} .
\end{aligned}
$$

5.5. Modulus of continuity. We will now show that $f$ has the required modulus of continuity. Let $x, y \in X$. We claim that there are points $w, z$ on the geodesic $L(x, y)$, connecting $x$ and $y$, such that $L(w, z) \subset A_{j, i}$ for some $j$ and $i$ and

$$
d(f(x), f(y)) \leq C d(f(w), f(z)) .
$$

We prove the claim only in the case where $x$ and $y$ are not in $C_{d}$, the general case is similar. By construction value of $f$ is constant in each component of the complement of $\bigcup_{j, i} A_{j, i}$. Therefore, if the points $x$ and $y$ lay outside the annuli we may choose points $x^{\prime \prime}, y^{\prime \prime} \in L(x, y)$ from some annuli such that $d(x, y) \geq d\left(x^{\prime \prime}, y^{\prime \prime}\right)$ and $|f(x)-f(y)|=\left|f\left(x^{\prime \prime}\right)-f\left(y^{\prime \prime}\right)\right|$. In what follows we assume that $x$ and $y$ are contained in annuli $A_{j_{x}, i_{x}}$ and $A_{j_{y}, i_{y}}$ respectively.

Let $x^{\prime}$ be the last point of $A_{j_{x}, i_{x}}$ on $L(x, y)$ and $y^{\prime}$ be the first point of $A_{j_{y}, i_{y}}$ on $L(x, y)$. Now consider $L\left(x^{\prime}, y^{\prime}\right)$. Denote by $J$ the set $\left\{A_{j, i}: d\left(x, a_{j, i}\right)<r_{j}\right.$ and $\left.d\left(y, a_{j, i}\right)>R_{j}\right\} \cup\left\{A_{j, i}: d\left(x, a_{j, i}\right)>R_{j}\right.$ and $\left.d\left(y, a_{j, i}\right)<r_{j}\right\}$ Obviously, $J$ contains at most two annuli of given generation. Moreover, for an annulus $A \in J$ we denote the first and last point of $L\left(x^{\prime}, y^{\prime}\right) \cap A$ by $x_{A}$ and $y_{A}$, respectively. Also, let $l=$ $\min \left\{j: A_{j, i} \in J\right\}$.

We also define $\mathcal{B}$ as the collection of all maximal balls $B_{j, i}$ which separate the geodesic $L\left(x^{\prime}, y^{\prime}\right)$ into two pieces. For a ball $B \in \mathcal{B}$ we denote the first and the last points of $L\left(x^{\prime}, y^{\prime}\right) \cap B$ by $x_{B}$ and $y_{B}$, respectively.

By definition of the mapping $f$, we have $d\left(f\left(x_{A}\right), f\left(y_{A}\right)\right) \leq C d_{j}$, whenever $A \in J$, and $d\left(f\left(x_{B}\right), f\left(y_{B}\right)\right)=0$, whenever $B \in \mathcal{B}$. The former follows from (48) and the latter from the fact that $f$ does not change its value on $\partial B\left(a_{j, i}, R_{j}\right)$. These give the estimate

$$
\begin{aligned}
d\left(f\left(x^{\prime}\right), f\left(y^{\prime}\right)\right) & \leq \sum_{A \in J} d\left(f\left(x_{A}\right), f\left(y_{A}\right)\right)+\sum_{B \in \mathcal{B}} d\left(f\left(x_{B}\right), f\left(y_{B}\right)\right) \\
& \leq \sum_{A \in J} C d_{j} \leq 2 C \sum_{j=l}^{\infty} d_{j} \leq 2 C d_{l} \sum_{j=0}^{\infty} 2^{-j} \leq C^{\prime} d_{l} .
\end{aligned}
$$

That is, $d\left(f\left(x^{\prime}\right), f\left(y^{\prime}\right)\right)$ is controlled by $C d_{l} \leq d(f(r), f(t))$, with $r, t \in L\left(x^{\prime}, y^{\prime}\right)$ points in some annulus of generation $l$. Finally,

$$
\begin{aligned}
d(f(x), f(y)) & \leq d\left(f(x), f\left(x^{\prime}\right)\right)+d\left(f\left(x^{\prime}\right), f\left(y^{\prime}\right)\right)+d\left(f\left(y^{\prime}\right), f(y)\right) \\
& \leq d\left(f(x), f\left(x^{\prime}\right)\right)+C d(f(r), f(t))+d\left(f\left(y^{\prime}\right), f(y)\right) .
\end{aligned}
$$

This implies the claim, since at least one of the terms on right hand side is at least $\frac{1}{3} d(f(x), f(y))$.

According to the previous claim we may assume that $x$ and $y$ are in the same annulus centered at $a$ with radii $r_{j}$ and $R_{j}$. Moreover, we may assume that $d(a, x) \leq$ 
$d(a, y)$. By definition,

$$
\begin{aligned}
|f(x)-f(y)| & \leq C d_{j}\left(\log \frac{R_{j}}{r_{j}}\right)^{-1}\left|\log \frac{d(x, a)}{d(y, a)}\right| \\
& =C d_{j}\left(\log \frac{R_{j}}{r_{j}}\right)^{-1}\left|\log \left(1+\frac{d(x, a)-d(y, a)}{d(y, a)}\right)\right| \\
& \leq C d_{j}\left(\log \frac{R_{j}}{r_{j}}\right)^{-1}\left|\log \left(1+\frac{d(x, y)}{r_{j}}\right)\right| .
\end{aligned}
$$

Choose a number $s$ such that $d(x, y)=u\left(2^{-s}\right)$. We consider two cases depending on the number $s$. If $j \leq s<j+1$, then $\log \left(1+\frac{d(x, y)}{r_{j}}\right) \leq \log \frac{2 d(x, y)}{r_{j}} \leq \log \frac{2^{n+1} R_{j}}{r_{j}}$. In this case (58) gives

$$
|f(x)-f(y)| \leq C d_{j} \leq C 2^{-j} \leq C 2^{-s} \leq C u^{-1}(d(x, y)) .
$$

This is the required modulus of continuity.

If $s>j+1$, then we have the following estimate for large enough $j$,

$$
\begin{aligned}
\frac{u\left(2^{-s}\right)}{u\left(2^{-j-1}\right)} & =\exp \left(\log \frac{1}{u\left(2^{-j-1}\right)}-\log \frac{1}{u\left(2^{-s}\right)}\right) \\
& \leq \exp \left(-\log 2 \frac{u^{\prime}\left(2^{-j-1}\right)}{u\left(2^{-j-1}\right)} 2^{-j-1}(s-j-1)\right) \leq 2^{j+1-s} .
\end{aligned}
$$

The last inequality follows from (9) and (3) for large $j$. Combing this with (58) we obtain

$$
\begin{aligned}
|f(x)-f(y)| & \leq C 2^{-j}\left|\log \left(1+\frac{d(x, y)}{r_{j}}\right)\right| \leq C 2^{-j} \frac{d(x, y)}{r_{j}} \leq C 2^{-j} 2^{j+1-s} \\
& \leq C 2^{-s} \leq C u^{-1}(d(x, y)) .
\end{aligned}
$$

This finishes the example.

\section{References}

[1] Davies, R. O.: Increasing sequences of sets and Hausdorff measure. - Proc. London Math. Soc. (3) 20, 1970, 222-236.

[2] Federer, H.: Geometric measure theory. - Grundlehren Math. Wiss. 153, Springer-Verlag New York Inc., New York, 1969.

[3] HajŁasz, P.: Sobolev spaces on an arbitrary metric space. - Potential Anal. 4:5, 1996, $403-415$.

[4] Heinonen, J., P. Koskela, N. Shanmugalingam, and J. T. Tyson: Sobolev classes of Banach space-valued functions and quasiconformal mappings. - J. Anal. Math. 85, 2001, 87139.

[5] Heinonen, J., P. Koskela, J. T. Tyson, and N. Shanmugalingam: Sobolev spaces on metric measure spaces: An approach based on upper gradients. - New Math. Monogr., Cambridge Univ. Press, 2015.

[6] Howroyd, J. D.: On the theory of Hausdorff measures in metric spaces. - PhD thesis, University College London, 1994.

[7] Jones, P. W., and N. G. Makarov: Density properties of harmonic measure. - Ann. of Math. (2), 142:3, 1995, 427-455.

[8] Kauranen, A., and P. Koskela,: Boundary blow up under Sobolev mappings. - Anal. PDE $7: 8,2014,1839-1850$. 
[9] Koskela, P., J. Malý, and T. ZÜrcher: Luzin's condition (N) and Sobolev mappings. - Atti Accad. Naz. Lincei Cl. Sci. Fis. Mat. Natur. Rend. Lincei (9) Mat. Appl. 23:4, 2012, 455-465.

[10] Koskela, P., and S. Rohde: Hausdorff dimension and mean porosity. - Math. Ann. 309:4, 1997, 593-609.

[11] LAAKso, T.J.: Ahlfors $Q$-regular spaces with arbitrary $Q>1$ admitting weak Poincaré inequality. - Geom. Funct. Anal. 10:1, 111-123.

[12] Mattila, P.: Geometry of sets and measures in Euclidean spaces. - Cambridge Stud. Adv. Math. 44, Cambridge Univ. Press, Cambridge, 1995.

[13] Nieminen, T.: Generalized mean porosity and dimension. - Ann. Acad. Sci. Fenn. Math. 31:1, 2006, 143-172.

Received 9 February 2015 • Accepted 22 May 2015 